\title{
Tratamientos pregerminativos para semillas de Prunus cercocarpifolia Villarreal (Rosales, Rosaceae), especie endémica de la Sierra Zapalinamé, Coahuila, México
}

\author{
Pregerminative seed treatments for Prunus cercocarpifolia Villarreal (Rosales, Rosaceae), \\ an endemic species from Sierra Zapalinamé, Coahuila, Mexico \\ David Castillo-Quiroz $^{1}$ (D) , Adriana Antonio-Bautista ${ }^{2}$ (D) Francisco Castillo-Reyes $^{1^{*}}$ (D), \\ Jesús Eduardo Sáenz-Ceja ${ }^{3}$ (D) , J. Trinidad Sáenz-Reyes ${ }^{4}$ (D)
}

\footnotetext{
${ }^{1}$ Campo Experimental Saltillo, Instituto Nacional de Investigaciones Forestales, Agrícolas y Pecuarias (INIFAP), Carretera Saltillo-Zacatecas km 342+119 No. 9515, Col. Hacienda de Buenavista, 25315, Saltillo, Coahuila, México.

${ }^{2}$ Programa de Doctorado en Recursos Fitogenéticos para Zonas Áridas, Departamento de Fitomejoramiento, Universidad Autónoma Agraria Antonio Narro, Calzada Antonio Narro No. 1923, Buenavista, 25315, Saltillo, Coahuila, México.

${ }^{3}$ Instituto de Investigaciones en Ecosistemas y Sustentabilidad, Universidad Nacional Autónoma de México, Antigua Carretera a Pátzcuaro No. 8701, Col. Ex Hacienda San José de la Huerta, 58190, Morelia, Michoacán, México.

${ }^{4}$ Campo Experimental Uruapan-INIFAP. Av. Latinoamericana No. 1101, Col. Revolución, 60500, Uruapan, Michoacán, México.

*Autor para correspondencia: reyes.francisco@inifap.gob.mx
}

Fecha de recepción:

27 de abril de 2020

Fecha de aceptación:

23 de agosto de 2020

Disponible en línea:

16 de marzo de 2021

Este es un artículo en acceso

abierto que se distribuye de

acuerdo a los términos de la

licencia Creative Commons.

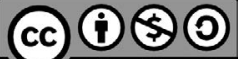

Reconocimiento-

NoComercial-

CompartirIgual 4.0

Internacional

\section{RESUMEN}

Prunus cercocarpifolia Villarreal (Rosaceae) es una especie arbustiva endémica, restringida a la Sierra Zapalinamé, en el sureste de Coahuila, México; sirve de refugio y alimento para fauna nativa, pero sus poblaciones han sido afectadas por actividades humanas. En este estudio, se evaluaron 11 tratamientos pregerminativos en esta especie, consistentes en la imbibición en agua y estratificación a diferentes tiempos y temperaturas. Se evaluó el porcentaje de semillas germinadas, duras y muertas, así como la proporción de plántulas normales y anormales. La imbibición de semillas con endocarpio por $48 \mathrm{~h}$ a $30^{\circ} \mathrm{C}$ logró el mejor porcentaje de germinación (89\%), ninguna semilla sin germinar y 58\% de plántulas normales. La estratificación por 8 días a $5^{\circ} \mathrm{C}$ logró ninguna semilla sin germinar y $64 \%$ de plántulas normales. La aplicación de estos tratamientos en la producción en vivero puede contribuir a la conservación de esta especie endémica del noreste de México.

\section{PALABRAS ClAVE}

Durazno cimarrón, endemismo, estratificación, imbibición, zonas semiáridas.

\section{ABSTRACT}

Prunus cercocarpifolia Villarreal (Rosaceae) is an endemic shrub species restricted to the Sierra Zapalinamé in southeastern Coahuila, Mexico. It is a refuge and food source for native fauna, but their populations have been affected by human activities. In this study, we evaluated 11 pregerminative treatments in seeds of this species. These treatments consisted of the imbibition in water and stratification at different times and temperatures. The treated seeds were sown, and we assessed the percentage of germinated, dark, and non-germinated seeds, as well as the proportion of normal and abnormal seedlings. The seed imbibition with endocarp for $48 \mathrm{~h}$ at $30^{\circ} \mathrm{C}$ reached the highest germination percentage (89\%), with all seeds being germinated, and $58 \%$ of normal seedlings produced. With the stratification for 8 days at $5^{\circ} \mathrm{C}$, all seeds were germinated and a $64 \%$ of normal seedlings was achieved. Implementing these treatments on nursery production can contribute to the conservation of this endemic species of northeastern Mexico.

\section{KEYWORDS}

Cimarron peach, endemism, stratification, imbibition, semiarid zones. 


\section{INTRODUCCIÓN}

Los ecosistemas forestales proveen una gran variedad de productos maderables y no maderables, así como servicios ambientales como la regulación climática y retención del suelo (Löf et al. 2016). Sin embargo, según la FAO (2015), la pérdida anual de superficie forestal mundial entre 2000 y 2010 alcanzó aproximadamente 7 millones de hectáreas, por efecto de actividades humanas, como la expansión de la frontera agrícola y ganadera, la construcción de caminos, el cambio de uso de suelo y la tala ilegal (Kuusela y Amacher 2016). La degradación de los bosques tiene severos impactos sobre la biodiversidad y, además, promueve el cambio climático (Löf et al. 2016). Algunas alternativas para mitigar estos impactos incluyen la delimitación de áreas naturales protegidas y corredores biológicos, el manejo forestal sustentable, sistemas agrosilvopastoriles y la restauración ecológica (Dudley et al. 2009; Retana-Guiascón et al. 2011). Una herramienta fundamental para la recuperación de las comunidades forestales es la reforestación (Cunningham et al. 2015), en la cuallas semillas desempeñan un papel muy importante en las acciones de conservación de las especies, pues son la fuente de germoplasma mayormente utilizado para la producción masiva de plantas; sin embargo, las especies silvestres no presentan una producción constante de las mismas, aunado a que en la mayoría se desconoce el mecanismo de regeneración natural (Villasana y Suárez 1997; Wightman y Santiago 2003).

Por ello, previo al establecimiento de plántulas producidas en vivero, es importante conocer los requerimientos físico-químicos y biológicos de las semillas para germinar y lograr una mejor producción de plántulas, ya que las semillas de la mayor parte de las plantas silvestres tienen un periodo de latencia (Valera y Arana 2011). Los tratamientos pregerminativos aplicados en semillas de estas especies permiten incrementar la producción en vivero, disminuir el tiempo y los costos de producción, así como lograr plántulas mejor adaptadas al trasplante en campo (Balaguera et al. 2010). Entre los tratamientos pregerminativos, destacan dos métodos: la imbibición en agua y la estratificación, los cuales contribuyen a mejorar el porcentaje de germinación y han sido probados en distintas especies agrícolas y forestales (Sánchez et al. 2001; Doria 2010; Ramírez et al. 2016).
En el norte de México, en el sureste del estado de Coahuila, se localiza la Sierra Zapalinamé, un área natural protegida donde confluyen el Desierto Chihuahuense y la Sierra Madre Oriental; está cubierta por distintos tipos de vegetación, que incluyen matorral xerófilo, bosque de encino, matorral submontano y bosque de coníferas, y alberga 78 especies vasculares (Encina-Domínguez et al. 2008). En esta región crece Prunus cercocarpifolia Villarreal (Rosaceae), localmente conocida como "durazno cimarrón", especie arbustiva que habita el matorral submontano, alcanza hasta $1.2 \mathrm{~m}$ de altura y llega a formar poblaciones densas en suelos pedregosos calcáreos o se asocia con otras especies arbustivas y pastizales (Villarreal-Quintanilla y Encinas-Domínguez 2005; Marines-Gómez 2016). El durazno cimarrón constituye el refugio y alimento para un gran número de especies de fauna silvestre, entre los que destacan el oso negro (Ursus americanus Pallas) y el perrito de la pradera (Cynemys mexicanus Merriam); sin embargo, actualmente, el sobrepastoreo de ganado, los incendios forestales provocados, la erosión y el ecoturismo desordenado disminuyen y fragmentan las poblaciones de esta especie arbustiva (Lagunes-Zavaleta 2015; Marines-Gómez 2018).

Por ello, para preservar el equilibrio ecológico y el funcionamiento del matorral submontano de la Sierra Zapalinamé, es necesaria la recuperación de las poblaciones del durazno cimarrón, a través de la reforestación. Sin embargo, en especies de Prunus silvestres se registran bajos porcentajes de germinación en ausencia de tratamientos pregerminativos que permitan la emergencia del embrión (Imani et al. 2011; Kim 2019). En el caso del durazno cimarrón, se desconoce si comparte esta característica y tampoco existe información sobre el efecto de tratamientos pregerminativos sobre las semillas de esta especie. Por ello, el objetivo de este estudio fue evaluar el efecto de 11 tratamientos pregerminativos sobre las semillas y la producción de plántulas de alta calidad de Prunus cercocarpifolia Villarreal.

\section{Materiales y Métodos}

\section{Origen de material biológico}

Se colectaron frutos maduros en el otoño del 2017, verificando que todas las semillas contaran con 
la madurez fisiológica adecuada, así como que estuvieran bien conformadas y en un estado masoso, pues esta última es una característica indicativa de que la semilla está completamente madura. La colecta se hizo en forma masal sobre 20 arbustos de P. cercocarpifolia, en una población natural que pertenece al tipo de vegetación matorral submontano del ejido Cuauhtémoc, en el municipio de Saltillo, Coahuila (25 16'16.62" N, 100 56'15.64" O, 2374 msnm) (Figura 1). El germoplasma fue trasladado al Campo Experimental Saltillo del Instituto Nacional de Investigaciones Forestales, Agrícolas y Pecuarias (INIFAP), para la extracción de las semillas. Posteriormente, fue enviado al Laboratorio de Producción de Semillas de la Universidad Autónoma Agraria Antonio Narro, donde se llevaron a cabo los tratamientos pregerminativos. cenamiento con temperatura controlada (Tipo Torrey). Se usaron cuatro réplicas de 15 semillas por cada tratamiento, para sumar un total de 660 semillas.

Las semillas pretratadas (Cuadro 1) fueron sembradas en macetas de plástico con sustrato húmedo a base de musgo (peat moss), con características sugeridas por la Asociación Internacional de Análisis de Semillas (Don y Ducournau 2018). Después de la siembra, éstas fueron colocadas en una cámara de germinación bioclimática (Barns tead/lab-line, modelo E82560N(, vwr International, Irving, Estados Unidos), bajo condiciones controladas de temperatura (26 \pm $2^{\circ} \mathrm{C}$ ) e iluminación (12 h:12 h luz/oscuridad), a partir de un diseño experimental completamente al azar. Se aplicaron cuatro riegos con agua destilada cada 4 días.

Veinte días después, a las semillas sembradas en

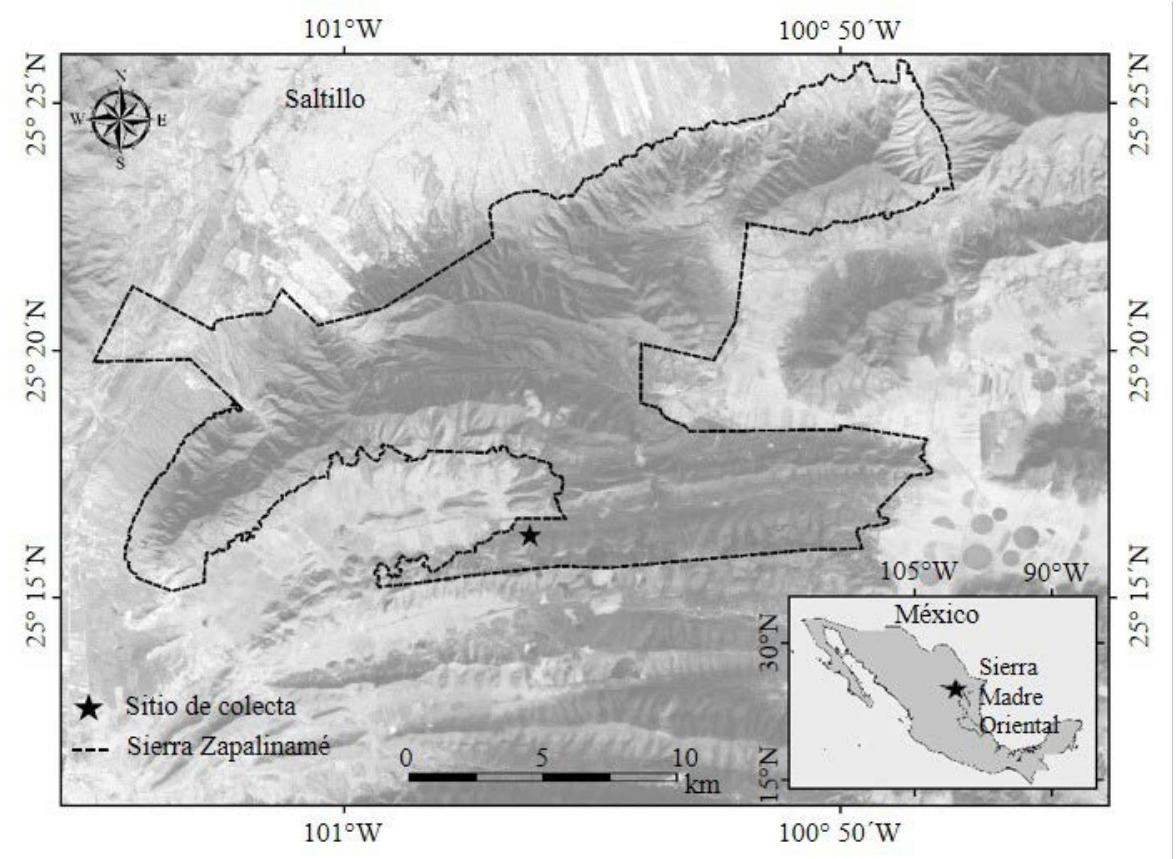

Figura 1. Localización del sitio de colecta de semillas de Prunus cercocarpifolia Villarreal en la Sierra Zapalinamé, parte de la Sierra Madre Oriental (SMO), noreste de México.

\section{Diseño experimental}

Semillaslimpiasfueronhomogenizadasmecánicamente con un divisor cónico tipo Boerner, y, posteriormente, fueron seleccionadas al azar. La imbibición consistió en colocar las semillas en un proceso de absorción de agua destilada a 5,25 y $30^{\circ} \mathrm{C}$, por diferentes intervalos de tiempo ( 24 o $48 \mathrm{~h}$ ), con y sin endocarpio. La estratificación se llevó a cabo sometiendo a las semillas a un proceso de enfriamiento a $5^{\circ} \mathrm{C}$, por $48 \mathrm{~h}$ u 8 días (Hartmann et al. 2010), utilizando cámaras de alma- las macetas se les evaluaron las siguientes variables: porcentaje de semillas germinadas (SG), para lo cual fueron consideradas todas aquellas semillas que presentaran $1 \mathrm{~mm}$ de radícula emitida; porcentaje de plántulas normales (PN), las cuales se definieron como la relación entre el número de semillas germinadas que tenían todas sus estructuras esenciales y el número de semillas plantadas; porcentaje de plántulas anormales (PA), se contaron aquellas plántulas que carecían de una o más de sus estructuras esenciales; porcentaje de semillas muertas (SM), el conteo incluyó todas aquellas semillas 
Cuadro 1. Tratamientos pregerminativos aplicados a semillas de durazno cimarrón (Prunus cercocarpifolia Villarreal).

\begin{tabular}{ll}
\hline Número & Tratamientos \\
\hline $\mathrm{T} 1$ & Control (semillas con endocarpio) \\
$\mathrm{T} 2$ & Control (semillas sin endocarpio) \\
$\mathrm{T} 3$ & Imbibición en agua por $48 \mathrm{~h}$ a $30^{\circ} \mathrm{C}$, semillas con endocarpio \\
$\mathrm{T} 4$ & Imbibición en agua por $24 \mathrm{~h}$ a $25^{\circ} \mathrm{C}$, semillas sin endocarpio \\
$\mathrm{T} 5$ & Imbibición en agua por $48 \mathrm{~h}$ a $25^{\circ} \mathrm{C}$, semillas sin endocarpio \\
T6 & Imbibición en agua por $48 \mathrm{~h}$ a $30^{\circ} \mathrm{C}$, semillas sin endocarpio \\
T7 & Imbibición en agua por $24 \mathrm{~h}$ a $30^{\circ} \mathrm{C}$, semillas sin endocarpio \\
T8 & Estratificación por 8 días a $5{ }^{\circ} \mathrm{C}$, semillas con endocarpio \\
T9 & Imbibición en agua por $48 \mathrm{~h}$ a $5{ }^{\circ} \mathrm{C}$, semillas con endocarpio \\
T10 & Imbibición en agua por $48 \mathrm{~h}$ a $5{ }^{\circ} \mathrm{C}$ seguido por $48 \mathrm{~h}$ a $30{ }^{\circ} \mathrm{C}$, semillas con endocarpio \\
T11 & Estratificación por $48 \mathrm{~h}$ a $5{ }^{\circ} \mathrm{C}$, semillas con endocarpio \\
\hline
\end{tabular}

que eran blandas una vez que absorbieron agua, pero no produjeron plántulas, y porcentaje de semillas duras (SD), se contaron todas las semillas que no absorbieron agua y que permanecieron impermeables al final del ensayo (Don 2013).

\section{Análisis estadístico}

El análisis estadístico de las variables SG, PN, PA, $\mathrm{SM}$ y SD se ejecutó con el lenguaje de programación $R$ versión 3.4.3 ( $R$ Core Team 2017). Se hizo la prueba Shapiro-Wilks para determinar la normalidad, así como una transformación de datos con Arcoseno $\sqrt{ }$ $(\mathrm{x}+1) / 100$, previo al análisis de varianza (función aov) y comparación de medias con pruebas de Tukey (función TukeyHSD), con un nivel de confianza de $95 \%$.

\section{Resultados Y discusión}

\section{Germinación de semillas}

La germinación de las semillas de P. cercocarpifolia varió entre 0 y $89 \%$, con diferencias significativas según el tratamiento aplicado (gl=10, F=14.955, P < 0.05) (Figura 2a). El menor porcentaje de germinación se encontró en los dos tratamientos control $(0 \%)$, mientras que el mayor porcentaje $(89 \%)$ se logró cuando las semillas se embebieron con endocarpio por $48 \mathrm{~h}$ a $30^{\circ} \mathrm{C}$ (T3) (Figura 2a). Las semillas con germinación mayor a $60 \%$ se lograron con estratificación con endocarpio por 8 días a $5^{\circ} \mathrm{C}(\mathrm{T} 8)$, imbibición de semillas sin endocarpio por $48 \mathrm{~h}$ a $30^{\circ} \mathrm{C}$ (T6), imbibición de semillas sin endocarpio por $24 \mathrm{~h}$ a $30^{\circ} \mathrm{C}$ (T7), imbibición de semillas con endocarpio por $48 \mathrm{~h}$ a $5^{\circ} \mathrm{C}$ (T10) e imbibición de semillas con endocarpio por $48 \mathrm{~h}$ a $25^{\circ} \mathrm{C}$ (T5).

En otras especies del género Prunus, la conservación del endocarpio limita la emergencia del embrión, mientras que, al quitarlo, se logran porcentajes de germinación altos (Chen et al. 2007; Imani et al. 2011). En este estudio, conservar el endocarpio no limitó la germinación de las semillas, lo cual puede representar menor tiempo dedicado a quitar el endocarpio, previo a la realización de los tratamientos. Además, permitió una germinación más homogénea en dichos tratamientos con respecto a aquéllos con porcentajes más bajos (Figura 2a).

El porcentaje de semillas duras fue bajo en la mayoría de los tratamientos (Figura 2b), excepto en los tratamientos control, donde se detectó $63 \%$ en semillas con endocarpio y $90 \%$ en semillas sin endocarpio. Se encontraron diferencias significativas entre los tratamientos evaluados ( $\mathrm{gl}=10, \mathrm{~F}=40.288, \mathrm{P}<0.05$ ); el tratamiento que difirió con respecto al resto fue la imbibición por $24 \mathrm{~h}$ a $30^{\circ} \mathrm{C}$ con endocarpio (T7), pues presentó 20\% de semillas duras (Figura $2 b$ ).

El porcentaje de semillas muertas mostró diferencias significativas entre tratamientos $(\mathrm{gl}=10$, $\mathrm{F}=7.9169, \mathrm{P}<0.05$ ) (Figura 2c); la estratificación por $48 \mathrm{~h}$ a $5^{\circ} \mathrm{C}$ con endocarpio (T11) con un porcentaje 
de $80 \%$, la imbibición por $48 \mathrm{~h}$ a $5^{\circ} \mathrm{C}$ con endocarpio (T9) de $72 \%$ y la imbibición por $24 \mathrm{~h}$ a $25^{\circ} \mathrm{C}$ sin endocarpio (T4) de $49 \%$ mostraron los porcentajes de semillas muertas; a su vez, estos tratamientos también presentaron las menores tasas de germinación. Al contrario, la imbibición en agua durante $24 \mathrm{~h}$ a $25^{\circ} \mathrm{C}$ con endocarpio (T3) y la estratificación por $8 \mathrm{~d}$ a $5^{\circ} \mathrm{C}$ con endocarpio (T8) no registraron semillas muertas, $\mathrm{y}$, a su vez, estos tratamientos presentaron las mayores tasas de germinación.

Los resultados sugieren que las semillas de P. cer-

(a)

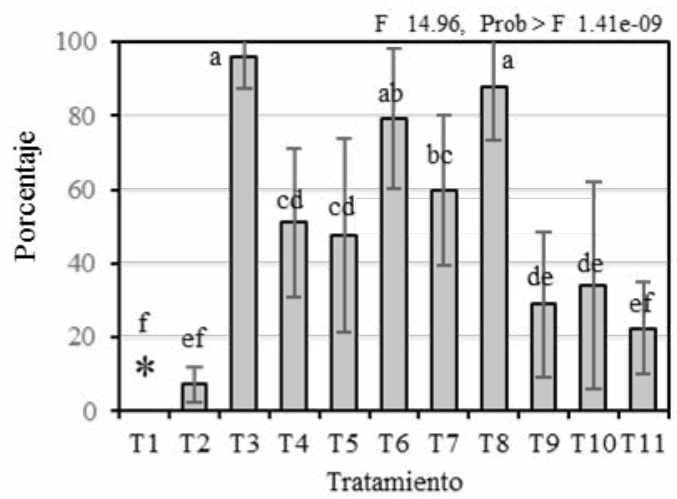

(b)

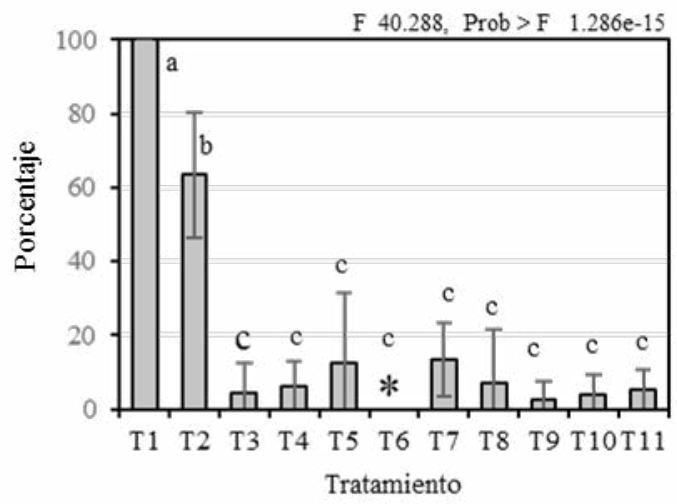

(c)

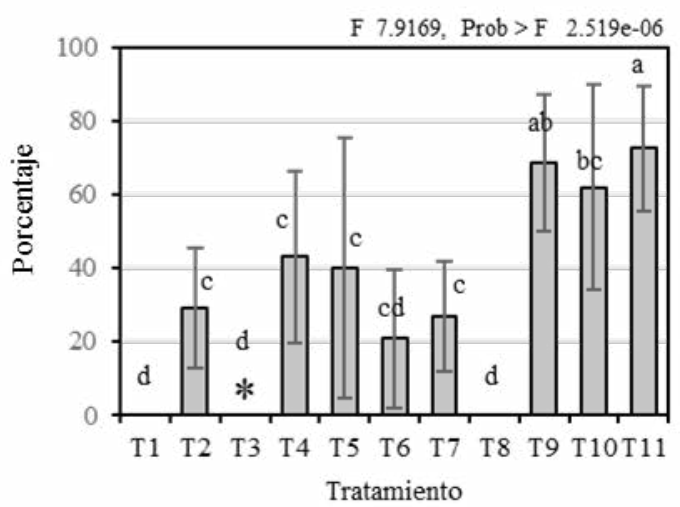

Figura 2. Porcentaje de semillas germinadas (a), duras (b) y muertas (c) de Prunus cercocarpifolia Villarreal, previa aplicación de tratamientos de imbibición y estratificación. Cada caja representa la media de 60 observaciones y su error estándar. cocarpifolia requieren acondicionamiento antes de ser propagadas en vivero. Esta característica es común en especies de Prunus y en diversas especies forestales de clima templado (Finch-Savage y Leubner-Metzger 2006; Imani et al. 2011). La imbibición y la estratificación mostraron una gran capacidad para lograr la germinación de las semillas de la especie de estudio, pero la imbibición en agua durante $24 \mathrm{~h} \mathrm{a} 25^{\circ} \mathrm{C}$ con endocarpio (T3) y estratificación por $8 \mathrm{~d}$ a $5^{\circ} \mathrm{C}$ con endocarpio (T8) destacaron por su eficiencia para aumentar la tasa de germinación. Es importante destacar que las semillas de durazno cimarrón requieren periodos de imbibición y estratificación cortos, pues largos periodos de inmersión pueden afectar la respiración celular y dañar las funciones vitales del embrión (Schmidt 2000; Barceló et al. 2009).

\section{Evaluación de calidad de plántula}

La proporción de plántulas normales difirió significativamente según el tratamiento aplicado $(\mathrm{gl}=10$, $\mathrm{F}=5.8991, \mathrm{P}<0.05)$. El porcentaje mayor de plántulas normales se obtuvo con la imbibición en agua durante $24 \mathrm{~h}$ a $25^{\circ} \mathrm{C}$ con endocarpio (T3), imbibición en agua por $48 \mathrm{~h}$ a $30^{\circ} \mathrm{C}$ sin endocarpio (T6) y estratificación por $8 \mathrm{~d}$ a $5^{\circ} \mathrm{C}$ con endocarpio (T8), mayor a $60 \%$. En contraste, los tratamientos control para los casos de imbibición en agua por $24 \mathrm{~h}$ a $25^{\circ} \mathrm{C}$ sin endocarpio (T4), imbibición en agua por $48 \mathrm{~h}$ a $25^{\circ} \mathrm{C}$ sin endocarpio (T5) e imbibición en agua por $48 \mathrm{~h}$ a $30^{\circ} \mathrm{C}$ sin endocarpio (T6) presentaron los menores porcentajes de plántulas normales (Figura 3a). Con respecto a las plántulas anormales, el porcentaje entre tratamientos varió entre 6 y 40\% con diferencias significativas ( $\mathrm{gl}=10, \mathrm{~F}=2.018$, $\mathrm{P}<0.05)$, donde la imbibición en agua durante $24 \mathrm{~h}$ a $25^{\circ} \mathrm{C}$ con endocarpio (T3), la imbibición en agua por $24 \mathrm{~h}$ a $25^{\circ} \mathrm{C} \sin$ endocarpio (T4) y la imbibición en agua por $48 \mathrm{~h}$ a $25^{\circ} \mathrm{C}$ sin endocarpio (T5) alcanzaron los mayores porcentajes (Figura $3 \mathrm{~b}$ ). Estos resultados indican que los tratamientos pregerminativos no sólo tienen efectos positivos sobre la germinación de las semillas, sino también efectos sobre la calidad de las plántulas, lo cual es relevante en la producción de plantas en vivero (Balaguera et al. 2010).

Los resultados de este estudio muestran que la imbibición y estratificación representan dos opciones viables para reducir la latencia de semillas de P. cerco- 
carpifolia y aumentar las tasas de germinación. Ambos han mostrado eficiencia en el aumento del porcentaje de germinación de especies como en Prunus domestica L. (De la Cruz et al. 2013), P. serotina Ehrh. (Phartyal et al. 2009) y P. persica L. (Bastch) (Imani et al. 2011). La imbibición con agua por $48 \mathrm{~h}$ a $30^{\circ} \mathrm{C}$ representa la

(a)

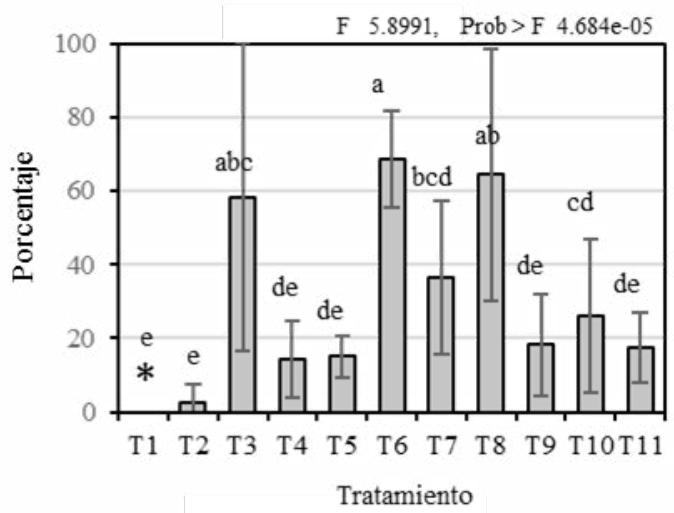

(b)

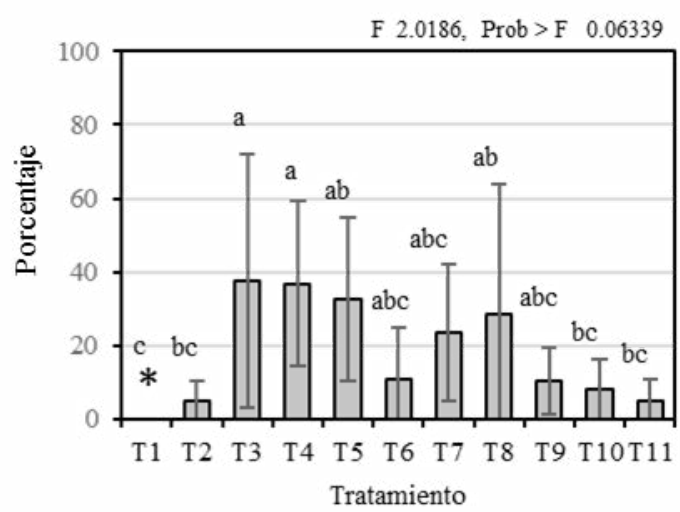

Figura 3. Porcentaje de plántulas normales (a) y anormales (b) de Prunus cercocarpifolia Villarreal, previa aplicación de tratamientos de imbibición y estratificación. Cada caja representa la media de 60 observaciones y su error estándar.

mejor opción para disminuir sustancialmente el tiempo destinado para procesar las semillas previo a su siembra en vivero, pues la estratificación a $5^{\circ} \mathrm{C}$ requiere 8 días de procesamiento. Además, la imbibición en agua es un método más sencillo en comparación con la inmersión de semillas con otras sustancias, como nitrógeno líquido y ácido sulfúrico, que mostró porcentajes de germinación similares en semillas de $P$. yedoensis Matsumura (Kim 2019).

Es importante evaluar el efecto de otros factores, como el sustrato, el tamaño y la procedencia de las semillas del durazno cimarrón, los cuales influyen sobre la emergencia de especies de zonas semiáridas (Vázquez et al. 2011; Rangel-Landa et al. 2015). La implementación de tratamientos pregerminativos sobre especies en riesgo ha sido exitosa en especies emparentadas como P. azorica (Hort. Ex Mouillef.) Rivas et al. (Moreira et al. 2012). Por ello, es fundamental implementar los tratamientos más exitosos en este estudio para aumentar las tasas de germinación, mejorar la producción en vivero con plántulas de buena calidad, y asegurar el establecimiento en campo del duraznillo cimarrón. Además, es necesario evaluar el efecto de tratamientos pregerminativos en otras especies forestales de importancia, para contribuir con mayor eficiencia a la recuperación de la Sierra Zapalinamé, área natural de gran importancia, debido a que provee más de $30 \%$ del agua potable que se consume en la ciudad de Saltillo, y, desde octubre de 1996, ha sido decretada con carácter de Zona Sujeta a Conservación Ecológica, por el Gobierno del Estado de Coahuila, con una ocupación de parte de los municipios de Saltillo y Arteaga (Marines-Gómez 2016).

\section{Conclusiones}

La germinación de las semillas de Prunus cercocarpifolia aumentó mediante la imbibición con agua por $48 \mathrm{~h}$ a $30^{\circ} \mathrm{C}$ con endocarpio y la estratificación por 8 días a $5^{\circ} \mathrm{C}$ con endocarpio. Estos tratamientos pregerminativos también tuvieron efectos positivos en la proporción de plántulas normales, por lo que se recomiendan ambas técnicas cuando se pretende propagar esta especie en vivero. La implementación de estos tratamientos pregerminativos y la reforestación con esta especie podrían contribuir a la recuperación del matorral submontano de la Sierra Zapalinamé, en el sureste de Coahuila.

\section{Agradecimientos}

Los autores agradecen a Juan Manuel Cárdenas Villanueva, Coordinador Operativo de la Reserva Natural Estatal Sierra de Zapalinamé, Profauna A.C., por su apoyo en la colecta del germoplasma. 


\section{LITERATURA CITADA}

Balaguera HE, Giovanni J, Cárdenas J. 2010. Efecto de la estratificación fría y la cobertura plástica en semillas de gulupa (Passiflora edulis Sims.) para la obtención de plántulas. Revista U.D.C.A Actualidad \& Divulgación Científica 13: 89-97. https://doi.org/10.31910/rudca.v13. n2.2010.735

Barceló CJ, Rodrigo GN, Sabater GB, Sánchez TR. 2009. Fisiología Vegetal. Ediciones Pirámide. Madrid, España. Chen S-Y, Chien C-T, Chung J-D, Yang Y-S, Kuo S-R. 2007. Dormancy-break and germination in seeds of Prunus campanulata (Rosaceae): Role of covering layers and changes in concentration of abscisic acid and gibberellins. Seed Science Research 27: 21-32. https://doi. org/10.1017/S0960258507383190

Cunningham SC, Nally RM, Baker PJ, Cavagnaro TR, Beringer J, Thompson JR, Thompson RM. 2015. Balancing the environmental benefits of reforestation in agricultural regions. Perspectives in Plant Ecology, Evolution and Systematics 17: 301-317. https://doi. org/10.1016/j.ppees.2015.06.001

De la Cruz J, López E, Zavaleta C, Mendoza W, Gonza A. 2013. Efecto de la estratificación en la germinación de semillas del ciruelo europeo, Prunus domestica. Rebiolest 1: 49-53.

Don R. 2013. ISTA Handbook on Seedling Evaluation. International Seed Testing Association. Bassersdorf, Suiza.

Don R, Ducournau S. 2018. Handbook ISTA on Seedling Evaluation. International Seed Testing Association. Bassersdorf, Suiza.

Doria J. 2010. Generalidades sobre las semillas: Su producción, conservación y almacenamiento. Cultivos Tropicales 31: 74-85.

Dudley N, Higgins-Zogib L, Mansourian S. 2009. The links between protected areas, faiths and sacred natural sites. Conservation Biology 23: 568-577. https://doi. org/10.1111/j.1523-1739.2009.01201.x

Encina-Domínguez JA, Encina-Domínguez FJ, MataRocha E, Valdés-Reyna J. 2008. Aspectos estructurales, composición florística y caracterización ecológica del bosque de oyamel de la Sierra de Zapalinamé, Coahuila, México. Boletín de la Sociedad Botánica de México 83: 13-24. https://doi.org/10.17129/botsci.1785

[FAO] Organización de las Naciones Unidas para la Alimentación y la Agricultura. [internet]. 2015.
Evaluación de los recursos forestales mundiales 2015. [cited 2020 marzo 20]. Roma, Italia. Disponible en: http:// www.fao.org/3/a-i4808s.pdf.

Finch-Savage WE, Leubner-Metzger G. 2006. Seed dormancy and the control of germination. New Phytologist 171: 501-523. https://doi.org/10.1111/j.1469-8137.2006.01787.x Hartmann HT, Kester D, Davies FT, Genéve RL. 2010. Hartmann and Kester's Plant Propagation: Principles and Practices. Pearson. Nueva York, Estados Unidos.

Imani A, Rasouli M, Tavakoli R, Zarifi E, Fatahi R, BarbaEspín G, Martínez-Gómez P. 2011. Optimization of seed germination in Prunus species combining hydrogen peroxide or gibberellic acid pre-treatment with stratification. Seed Science and Technology 39: 204-207. https:// doi.org/10.15258/sst.2011.39.1.18

Kim DH. 2019. Practical methods for rapid seed germination from seed coat-imposed dormancy of Prunus yedoensis. Scientia Horticulturae 243: 451-456. https://doi. org/10.1016/j.scienta.2018.08.039

Kuusela O-P, Amacher GS. 2016. A review of performance bonding in forest policy settings. Current Forestry Reports 2: 189-200. https://doi.org/10.1007/ s40725-016-0043-y

Lagunes-Zavaleta SE. 2015. Ecología y abundancia del almendrillo Silvestre (Prunus cercocarpifolia Villarreal), especie endémica del sureste de Coahuila. Tesis profesional. Universidad Autónoma Agraria Antonio Narro. Saltillo, México.

Löf M, Brunet J, Filyushkina A, Lindbladh M, Skovsgaard JP, Felton A. 2016. Management of oak forests: Striking a balance between timber production, biodiversity and cultural services. International Journal of Biodiversity Science, Ecosystem Services and Management 12: 59-73. https://doi.org/10.1080/21513732.2015.1120780

Marines-Gómez SC. [internet]. 2016. A 20 años de nuevos hallazgos y nuevos retos: Zona Sujeta a Conservación Ecológica Sierra de Zapalinamé. Secretaría de Medio Ambiente de Coahuila. Saltillo, México. [cited 2020 enero 14]. Disponible en: https://www.sema.gob.mx/descargas/manuales/Bordeando_36.pdf

Marines-Gómez SC. [internet]. 2018. El Aguaje, Programa Especial de Manejo. Secretaría de Medio Ambiente de Coahuila. Saltillo, México. [cited 2020 enero 14]. Disponible en: http://www.coahuilatransparente.gob. $\mathrm{mx} /$ disp/documentos_disp/SEMA.\%20Programa\%20 de\%20Manejo\%20SAN\%20LORENZO.pdf

Moreira OCB, Martins J, Silva L, Moura M. 2012. Seed germi- 
nation and seedling growth of the endangered Azorean cherry Prunus azorica. HortScience 47: 1222-1227. https:// doi.org/10.21273/HORTSCI.47.9.1222

Phartyal SS, Godefroid S, Koedam N. 2009. Seed development and germination ecophysiology of the invasive tree Prunus serotina (Rosaceae) in a temperate forest in Western Europe. Plant Ecology 204: 285-294. https://doi. org/10.1007/s11258-009-9591-6

R Core Team. [internet]. 2017. R: A language for statistical analysis. Viena, Austria. Disponible en: https://www.r-project.org/

Ramírez HM, Niño R, Aguirre JR, Flores J, De-Nova JA, Jarquin R. 2016. Seed viability and effect of temperature on germination of Agave angustifolia subsp. tequilana and A. mapisaga; two useful Agave species. Genetic Resources and Crop Evolution 63: 881-888. https://doi.org/10.1007/ s10722-015-0291-x

Rangel-Landa S, Casas A, Dávila P. 2015. Facilitation for Agave potatorum: An ecological approach for assisted population recovery. Forest Ecology and Management 347: 57-74. https://doi.org/10.1016/j.foreco.2015.03.003

Retana-Guiascón O, Aguilar-Nah M, Niño-Gómez G. 2011. Uso de la vida silvestre y alternativas de manejo integral. El caso de la comunidad Maya de Pich, Campeche, México. Tropical and Subtropical Agroecosystems 14: 885-890.

Sánchez JA, Orta R, Muñoz BC. 2001. Tratamientos pregerminativos de hidratación-deshidratación de las semillas y sus efectos en plantas de interés agrícola. Agronomía Costarricense 25: 67-91.

Schmidt L. 2000. Guide to Handling of Tropical and Subtropical Forest Seed. Danida Forest Seed Center. Humlebaek, Dinamarca.

Valera SA, Arana V. [internet]. 2011. Silvicultura en vivero. Instituto Nacional de Tecnología Agropecuaria. Bariloche, Argentina. [cited 2019 diciembre 28]. Disponible en: https://inta.gob.ar/sites/default/files/ script-tmp-inta_latencia.pdf.

Vázquez E, García JR, Peña CB, Ramírez HM, Morales V. 2011. Tamaño de la semilla, emergencia y desarrollo de la plántula de maguey (Agave salmiana Otto ex SalmDyck). Revista Fitotecnia Mexicana 34: 167-173.

Villarreal-Quintanilla JA, Encinas-Domínguez JA. 2005. Plantas vasculares endémicas de Coahuila y algunas áreas adyacentes, México. Acta Botánica Mexicana 70: 1-46. https://doi.org/10.21829/abm70.2005.986

Villasana ARA, Suárez A. 1997. Estudio fenológico de die- ciséis especies forestales presentes en la reserva forestal Imataca estado Bolívar-Venezuela. Revista Forestal Venezolana 41: 13-21.

Wightman KE, Santiago B. 2003. La cadena de la reforestación y la importancia en la calidad de las plantas. Foresta Veracruzana 5: 45-51. 